\title{
Occupational Patterns of Opioid-Related Overdose Deaths Among Arizona Medicaid Enrollees, 2008-2017
}

\author{
Rohan Chalasani ', Wei-Hsuan Lo-Ciganic, MS, PhD'2, James L. Huang, PhD', \\ Jingchuan Guo, PhD, MD, MPH' , Jeremy C. Weiss, MD, PhD ${ }^{3}$, \\ Courtney C. Kuza, PhD, MPH' and Walid F. Gellad, MD, MPH ${ }^{7,4}$
}

\begin{abstract}
'Center for Pharmaceutical Policy and Prescribing, Health Policy Institute, University of Pittsburgh, Pittsburgh, PA, USA; ${ }^{2}$ Department of Pharmaceutical Outcomes and Policy and Center for Drug Evaluation and Safety, University of Florida, Gainesville, FL, USA; ${ }^{3} \mathrm{Heinz}$ College, Carnegie Mellon University, Pittsburgh, PA, USA; ${ }^{4}$ Department of General Internal Medicine, School of Medicine, University of Pittsburgh, Pittsburgh, PA, USA.
\end{abstract}

J Gen Intern Med 35(7):2210-3

DOI: $10.1007 / \mathrm{s} 11606-020-05684-7$

(c) Society of General Internal Medicine (This is a U.S. government work and not under copyright protection in the U.S.; foreign copyright protection may apply) 2020

\section{INTRODUCTION}

Nearly 400,000 individuals died from opioid overdose in the USA from 1999 to $2017 .{ }^{1}$ Understanding the populations most affected is crucial to developing targeted interventions. Prior studies examining occupational patterns of opioid-related overdose deaths were limited because they did not examine unpaid occupations potentially relevant to opioid overdose (e.g., homemakers, students, unemployed) or changes in occupational patterns over time. ${ }^{2-4}$ We aimed to more comprehensively examine occupational patterns of opioid-related overdose deaths among Arizona Medicaid enrollees with opioid prescriptions from 2008 to 2017. Over that time period, opioid-related overdose deaths in Arizona sharply increased from 586 in 2008 to 949 in $2017 .^{5}$

\section{METHODS}

We identified Arizona Medicaid enrollees aged 18-64 years who filled any opioid prescriptions and died from accidental opioidrelated overdose from 2008 to 2017. We identified opioid-related overdose deaths in death certificates using International Classification of Diseases, Tenth Revision (ICD-10) underlying cause-ofdeath codes X42, X44, Y12, and Y14 for accidental and undetermined overdose and multiple cause-of-death codes T40.1 (heroin), T40.2 (natural and semisynthetic opioids), T40.3 (methadone), and T40.4 (synthetic opioids other than methadone).

We extracted age, year of death, occupation, gender, race, overdose type (heroin-related, synthetic opioid-related), and co-morbid drug use (other substances involved in the

Received September 18, 2019

Accepted January 26, 2020

Published online February 10, 2020 overdose) from death certificates. We classified individuals' occupations based on the Bureau of Labor Statistics' 2018 Standard Occupational Classification (SOC) system. We created four additional occupational categories not part of the classification system: homemakers; caregivers; students and volunteers; and unemployed, never worked, and disabled. For each occupational group, we calculated the number of opioidrelated overdose deaths in each year and described demographic characteristics and proportion with heroin-related overdose deaths, synthetic opioid-related deaths, and comorbid drug use.

\section{RESULTS}

From 2008 to 2017, 2339 eligible Arizona Medicaid enrollees died from opioid-related overdose, with 644 unique occupations and 148 individuals of unknown occupation. Overall, the mean age at death was $41(\mathrm{SD}=12)$ years, with $58 \%$ male, $66 \%$ White, $15 \%$ Hispanic, 32\% involving heroin, $13 \%$ involving synthetic opioids, and $40 \%$ involving co-morbid drug use (Table 1).

The gender and race distribution and proportion with heroin-related death varied substantially across occupations. Males were especially predominant among construction and extraction (>95\%); installation, maintenance, and repair (> 95\%); and architecture and engineering occupations, but minimal among homemakers, healthcare support occupations, and caregivers. Non-Hispanic Whites were prominent in overdose deaths among computer and mathematical $(88 \%)$ and business and financial operations (81\%) occupations, but low among caregivers $(38 \%)$. Of all opioid-related overdose deaths, heroin-related deaths were more common among construction and extraction occupations (42\%); arts, design, entertainment, sports, and media (40\%); and building and grounds cleaning occupations (50\%), but uncommon in occupations such as homemakers, business/financial, and legal occupations (Table 1). The percentage with non-opioid co-morbid drugs involved in the cause of death was between 32 and $46 \%$ across occupations. 
Table 1 Opioid-related overdose deaths among Arizona Medicaid enrollees with opioid prescriptions, mean age at death, gender and race distribution, and proportion of heroin-related overdose deaths, by occupational group, 2008-2017

\begin{tabular}{|c|c|c|c|c|c|c|c|c|}
\hline $\begin{array}{l}\text { Occupational } \\
\text { Group }\end{array}$ & $\begin{array}{l}\text { Representative } \\
\text { occupations }\end{array}$ & $\begin{array}{l}\text { Total } \\
\text { opioid- } \\
\text { related } \\
\text { overdose } \\
\text { deaths }\end{array}$ & $\begin{array}{l}\text { Mean } \\
\text { age at } \\
\text { death } \\
\text { (SD) }\end{array}$ & $\begin{array}{l}\% \\
\text { Male }\end{array}$ & $\begin{array}{l}\text { \% Non- } \\
\text { Hispanic } \\
\text { White }\end{array}$ & $\begin{array}{l}\% \text { Heroin- } \\
\text { related } \\
\text { overdose } \\
\text { deaths }\end{array}$ & $\begin{array}{l}\text { \% Synthetic } \\
\text { opioid-related } \\
\text { overdose deaths } \\
\text { (except } \\
\text { methadone) }\end{array}$ & $\begin{array}{l}\text { \% Co- } \\
\text { morbid, } \\
\text { non-opioid } \\
\text { drug use }\end{array}$ \\
\hline Homemakers* & $\begin{array}{l}\text { homemaker, } \\
\text { housewife }\end{array}$ & 262 & $42(11)$ & $\dagger$ & 58 & 16 & 14 & 38 \\
\hline $\begin{array}{l}\text { Construction and } \\
\text { Extraction }\end{array}$ & $\begin{array}{l}\text { brickmason, } \\
\text { roofer }\end{array}$ & 233 & $44(12)$ & $>95$ & 66 & 42 & 9 & 44 \\
\hline $\begin{array}{l}\text { Transportation and } \\
\text { Material Moving }\end{array}$ & $\begin{array}{l}\text { bus driver, } \\
\text { general laborer }\end{array}$ & 197 & $42(12)$ & 91 & 56 & 40 & 13 & 40 \\
\hline Sales and Related & $\begin{array}{l}\text { cashier, sales } \\
\text { agent }\end{array}$ & 179 & $40(12)$ & 61 & 68 & 31 & 15 & 43 \\
\hline $\begin{array}{l}\text { Food Preparation } \\
\text { and Serving } \\
\text { Related }\end{array}$ & chef, waiter & 166 & $39(12)$ & 50 & 75 & 33 & 14 & 44 \\
\hline $\begin{array}{l}\text { Office and } \\
\text { Administrative } \\
\text { Support }\end{array}$ & $\begin{array}{l}\text { customer service, } \\
\text { teller }\end{array}$ & 139 & $41(12)$ & 32 & 62 & 24 & 14 & 35 \\
\hline Management & $\begin{array}{l}\text { chief executive, } \\
\text { hotel manager }\end{array}$ & 131 & $42(11)$ & 66 & 69 & 31 & 12 & 36 \\
\hline $\begin{array}{l}\text { Installation, } \\
\text { Maintenance, and } \\
\text { Repair }\end{array}$ & $\begin{array}{l}\text { car mechanic, } \\
\text { locksmith }\end{array}$ & 127 & $43(11)$ & $>95$ & 71 & 40 & 11 & 39 \\
\hline $\begin{array}{l}\text { Unemployed, } \\
\text { Never Worked, } \\
\text { Disabled* }\end{array}$ & $\begin{array}{l}\text { disabled, } \\
\text { unemployed }\end{array}$ & 102 & $36(12)$ & 63 & 55 & 39 & 12 & 44 \\
\hline $\begin{array}{l}\text { Students, } \\
\text { Volunteers* }\end{array}$ & $\begin{array}{l}\text { college student, } \\
\text { volunteer }\end{array}$ & 88 & $27(8)$ & 49 & 65 & 40 & 13 & 40 \\
\hline $\begin{array}{l}\text { Healthcare } \\
\text { Practitioners and } \\
\text { Technical }\end{array}$ & $\begin{array}{l}\text { physician, } \\
\text { registered nurse }\end{array}$ & 72 & 44 (10) & 33 & 79 & $\dagger$ & 21 & 32 \\
\hline Healthcare Support & $\begin{array}{l}\text { dental assistant, } \\
\text { orderly }\end{array}$ & 69 & $43(11)$ & $\dagger$ & 71 & 22 & 16 & 36 \\
\hline Production & butcher, machinist & 64 & $45(12)$ & 83 & 59 & 41 & $\dagger$ & 42 \\
\hline $\begin{array}{l}\text { Arts, Design, } \\
\text { Entertainment, } \\
\text { Sports, and Media }\end{array}$ & $\begin{array}{l}\text { actress, } \\
\text { photographer }\end{array}$ & 62 & $42(12)$ & 74 & 77 & 44 & $\dagger$ & 40 \\
\hline $\begin{array}{l}\text { Building and } \\
\text { Grounds Cleaning } \\
\text { and Maintenance }\end{array}$ & $\begin{array}{l}\text { custodian, } \\
\text { landscaper }\end{array}$ & 58 & $43(13)$ & 71 & 53 & 50 & $\dagger$ & 43 \\
\hline $\begin{array}{l}\text { Personal Care and } \\
\text { Service }\end{array}$ & $\begin{array}{l}\text { barber, personal } \\
\text { trainer }\end{array}$ & 41 & $38(12)$ & 29 & 68 & 29 & $\dagger$ & 41 \\
\hline Caregivers* & $\begin{array}{l}\text { caregiver, } \\
\text { caretaker }\end{array}$ & 37 & $44(13)$ & $\dagger$ & 38 & $\dagger$ & $\dagger$ & 43 \\
\hline $\begin{array}{l}\text { Community and } \\
\text { Social Service }\end{array}$ & $\begin{array}{l}\text { priest, social } \\
\text { worker }\end{array}$ & 36 & $41(12)$ & 58 & 67 & 39 & $\dagger$ & 39 \\
\hline $\begin{array}{l}\text { Business and } \\
\text { Financial } \\
\text { Operations }\end{array}$ & $\begin{array}{l}\text { accountant, loan } \\
\text { officer }\end{array}$ & 32 & $43(8)$ & 56 & 81 & $\dagger$ & $\dagger$ & 34 \\
\hline Protective Service & $\begin{array}{l}\text { firefighter, } \\
\text { security guard }\end{array}$ & 24 & $42(12)$ & $\dagger$ & 79 & $\dagger$ & $\dagger$ & 46 \\
\hline $\begin{array}{l}\text { Educational } \\
\text { Instruction and } \\
\text { Library }\end{array}$ & $\begin{array}{l}\text { curator, high } \\
\text { school teacher, }\end{array}$ & 19 & $43(10)$ & $\dagger$ & 68 & $\dagger$ & $\dagger$ & $\dagger$ \\
\hline $\begin{array}{l}\text { Computer and } \\
\text { Mathematical }\end{array}$ & $\begin{array}{l}\text { data architect, } \\
\text { web developer }\end{array}$ & 16 & $40(9)$ & $\dagger$ & 88 & $\dagger$ & $\dagger$ & $\dagger$ \\
\hline $\begin{array}{l}\text { Architecture and } \\
\text { Engineering }\end{array}$ & $\begin{array}{l}\text { architect, } \\
\text { electrical engineer }\end{array}$ & $\dagger$ & $46(11)$ & $\dagger$ & $\dagger$ & $\dagger$ & $\dagger$ & $\dagger$ \\
\hline Legal & $\begin{array}{l}\text { attorney, legal } \\
\text { assistant }\end{array}$ & $\dagger$ & $51(7)$ & $\dagger$ & $\dagger$ & $\dagger$ & $\dagger$ & $\dagger$ \\
\hline $\begin{array}{l}\text { Farming, Fishing, } \\
\text { and Forestry }\end{array}$ & $\begin{array}{l}\text { combine operator, } \\
\text { farmworker }\end{array}$ & $\dagger$ & $40(7)$ & $\dagger$ & $\dagger$ & $\dagger$ & $\dagger$ & $\dagger$ \\
\hline Military & $\begin{array}{l}\text { artillery officer, } \\
\text { infantryman }\end{array}$ & $\dagger$ & $36(12)$ & $\dagger$ & $\dagger$ & $\dagger$ & $\dagger$ & $\dagger$ \\
\hline $\begin{array}{l}\text { Life, Physical, and } \\
\text { Social Science }\end{array}$ & $\begin{array}{l}\text { economist, } \\
\text { physicist }\end{array}$ & $\dagger$ & $50(3)$ & $\dagger$ & $\dagger$ & $\dagger$ & $\dagger$ & $\dagger$ \\
\hline
\end{tabular}


Table 1. (continued)

\begin{tabular}{llllllll}
\hline \hline $\begin{array}{l}\text { Occupational } \\
\text { Group }\end{array}$ & $\begin{array}{l}\text { Representative } \\
\text { occupations }\end{array}$ & $\begin{array}{l}\text { Total } \\
\text { opioid- } \\
\text { related } \\
\text { overdose } \\
\text { deaths }\end{array}$ & $\begin{array}{l}\text { Mean } \\
\text { age at } \\
\text { death } \\
\text { (SD) }\end{array}$ & $\begin{array}{l}\text { \% } \\
\text { Male }\end{array}$ & $\begin{array}{l}\text { \% Non- } \\
\text { Hispanic } \\
\text { White* }\end{array}$ & $\begin{array}{l}\text { \% Heroin- } \\
\text { related } \\
\text { overdose } \\
\text { deaths }\end{array}$ & $\begin{array}{l}\text { \% Synthetic } \\
\text { opioid-related } \\
\text { overdose deaths } \\
\text { (except } \\
\text { methadone) }\end{array}$ \\
\hline $\begin{array}{l}\text { Unknown } \\
\begin{array}{l}\text { All Opioid } \\
\text { Overdose Deaths }\end{array}\end{array}$ & - & 148 & $48(10)$ & 71 & 73 & 42 & $\begin{array}{l}\text { \% Co- } \\
\text { morbid, } \\
\text { non-opioid } \\
\text { drug use** }\end{array}$ \\
\hline
\end{tabular}

Data Source: Arizona Department of Health Services and Arizona Health Care Cost Containment System, 2019. Note: The Center for Health Information \& Research is the source for all processing of the ADHS and AHCCCS data.

*Occupational group is a non-standard group added to the Bureau of Labor Statistics 2018 Standard Occupational Classification.

**Co-morbid drug use was identified by T codes corresponding to cocaine (T40.5), psychostimulants (T43.6), benzodiazepines (T42.4), cannabis (T40.7), lysergide (T40.8), or other psychodyspleptics (T40.9), as indicated in the cause of death in death certificates.

†Data suppressed due to insufficient cell size.

tOther races not presented due to insufficient cell size. Blacks were 4\%, Hispanics were 15\%, and Native Americans were $3 \%$ of all opioid overdoserelated deaths

Figure 1 shows the occupations with the most opioidrelated overdose deaths from 2008 to 2017. Overall, opioidrelated overdose deaths were consistently high among construction and extraction workers and homemakers over time. The number of opioid-related overdose deaths increased in recent years among transportation and material moving occupations, the group with the most deaths in $2017(n=51)$.] $->$

\section{DISCUSSION}

This study offers unique demographic insights into opioidrelated overdose deaths among Medicaid enrollees with opioid prescriptions. We identified a previously unexamined occupational group - homemakers - that is consistently among the highest levels of opioid-related overdose deaths in this population. Additionally, by tracking occupational patterns year-toyear, we identified the recent rapid increase in the number of opioid-related overdose deaths among transportation and material moving occupations.

Our study has several limitations. First, occupations were identified from death certificates without further validation. Second, we are not able to examine whether observed patterns were associated with changes in Medicaid enrollment, occupational distributions among Medicaid enrollees, or opioid prescribing patterns. Finally, our findings focus on Arizona

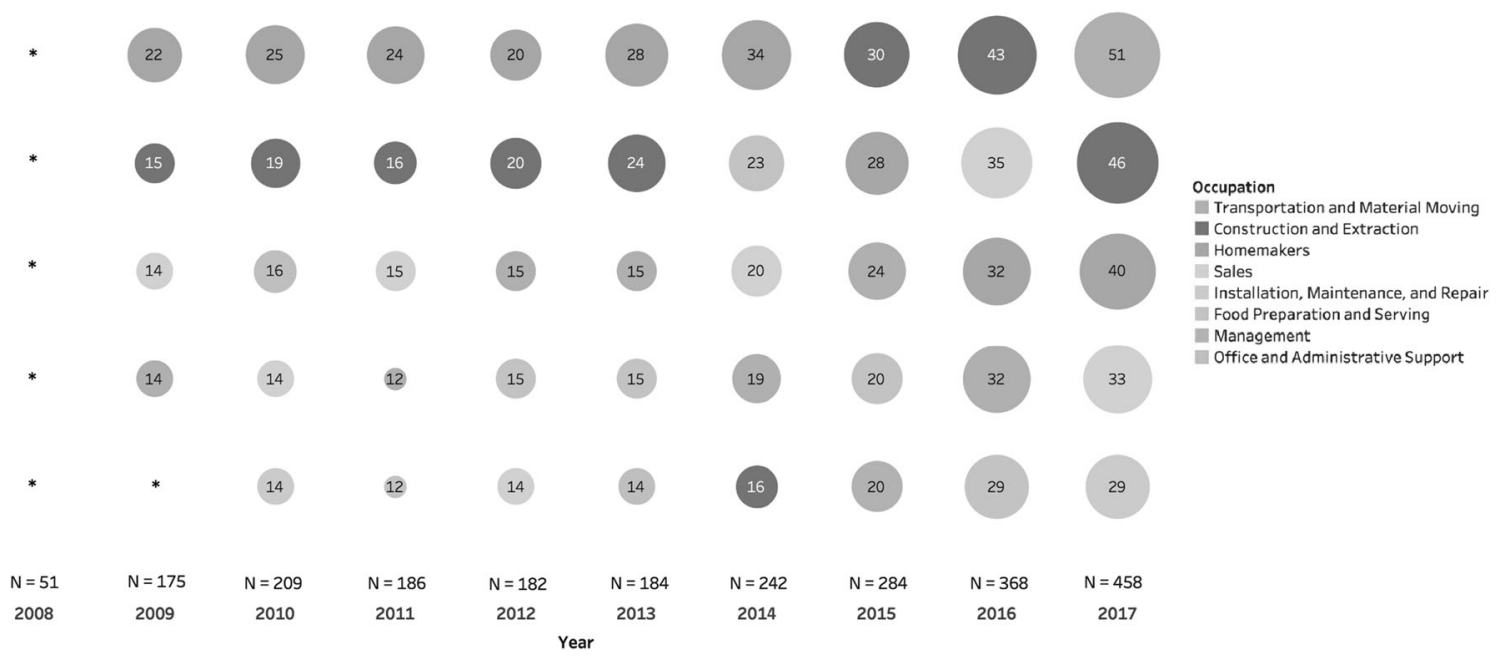

Fig. 1 Number of opioid-related overdose deaths among Arizona Medicaid enrollees with opioid prescriptions, by occupational group and year. The top 5 occupations each year are listed, 2008-2017. Data Source: Arizona Department of Health Services and Arizona Health Care Cost Containment System, 2019. Note: The Center for Health Information and Research is the source for all processing of the ADHS and AHCCCS data. The number in each circle represents the number of opioid-related overdose deaths for that occupation in a given year. Unknown occupations were excluded from this ranking

*Data on number of opioid-related overdose deaths was suppressed for all occupations in 2008 and management occupations in 2009 due to insufficient cell size 
Medicaid beneficiaries with opioid prescriptions and have limited generalizability to other populations.

Nevertheless, these findings identify several occupational groups strongly affected by fatal opioid overdose and offer additional demographic insight that could inform targeted interventions, from targeting naloxone distribution to work sites of high-risk occupations, to increasing licensing or educational requirements around opioids in certain fields, to additional interventions that might target those unpaid occupations we identify in this study.

Corresponding Author: Walid F. Gellad, MD, MPH; Department of General Internal Medicine, School of MedicineUniversity of Pittsburgh, Pittsburgh, PA, USA (e-mail: walid.gellad@pitt.edu).

Funding Information This study was supported by grant RO1DA044985 from the NIH/National Institute on Drug Abuse and by grant R21AG060308 from the NIH/National Institute of Aging.

\section{Compliance with Ethical Standards:}

Conflict of Interest: The authors declare that they do not have a conflict of interest.

Publisher's Note: Springer Nature remains neutral with regard to jurisdictional claims in published maps and institutional affiliations.

\section{REFERENCES}

1. Scholl L, Seth P, Kariisa M, Wilson N, Baldwin G. Drug and opioidinvolved overdose deaths-United States, 2013-2017. MMWR Morb Mortal Wkly Rep. 2019; 67: 1419-1427. https://doi.org/10.15585/mmwr. mm675152el

2. Morano LH, Steege AL, Luckhaupt SE. Occupational patterns in unintentional and undetermined drug-involved and opioid-involved overdose deaths-United States, 2007-2012. MMWR Morb Mortal Wkly Rep. 2018; 67: 925-930. doi: https://doi.org/10.15585/mmwr.mm6733a3

3. Massachusetts Department of Public Health. Opioid-related overdose deaths in Massachusetts by industry and occupation, 2011-2015. https://www.mass.gov/files/documents/2018/08/15/opioid-industryoccupation.pdf. Published August 7, 2018. Accessed 15 Aug 2019.

4. Utah Department of Health. Opioid Deaths by Occupation Groups in Utah 2012-2017. 2019. https://ibis.health.utah.gov/ibisph-view/pdf/opha/ publication/hsu/2019/1902_OpioidOcc.pdf. Published February 4, 2019. Accessed 15 Aug 2019.

5. Arizona Department of Health Services. Arizona Opioid Emergency Response, June 2017 to June 2018. https://www.azdhs.gov/documents/prevention/womens-childrens-health/injury-prevention/opioidprevention/2017-opioid-emergency-response-report.pdf. Published June 2018. Accessed 3 Sept 2019.

Publisher's Note Springer Nature remains neutral with regard to jurisdictional claims in published maps and institutional affiliations. 